Pacific Journal of Mathematics

DÉRIVATIONS, ET HAUTES DÉRIVATIONS, DANA CERTAIN Dumas aND ROBert VIDAL 


\title{
DERIVATIONS, ET HAUTES DERIVATIONS, DANS CERTAINS CORPS GAUCHES DE SERIES DE LAURENT
}

\author{
Francois Dumas et Robert Vidal
}

Let $k$ be a field of characteristic zero, $F=k((Y))$ the local field of Laurent series in a variable $Y$, and $\partial_{Y}$ the usual derivation of $F$. The purpose of this paper is the complete description of $k$-derivations on the formal pseudo-differential operator skewfield $K=F\left(\left(X, \partial_{Y}\right)\right)$. An application of this result is given to study the structure of higher derivations in skewfields of characteristic zero.

\section{Présentation du problème.}

1.1. Anneaux de Cohen et hautes dérivations. Les anneaux de valuation discrète complets non commutatifs et les corps gauches locaux (qui sont leurs corps de fractions) ont été intensément étudiés au cours des dernièrnes années (cf. bibliographie). Un outil essentiel pour cette étude est la notion de haute $\sigma$-dérivation au sens de P. M. Cohn (cf. [2]), d'un corps non nécessairement commutatif. Dans cet article, comme en [1], [3], [4], [5], [6], [10], [11], [12], on s'intéresse plus particulièrement aux hautes dérivations, c'est-à-dire au cas où $\sigma$ est l'application identique. Rappelons-en la définition: une haute dérivation d'un corps non nécessairement commutatif $K$ est une suite $S=\left(\delta_{n}\right)_{n \geq 0}$ d'applications additives de $K$ dans $K$, avec $\delta_{0}=\mathrm{id}_{K}$, telle que l'on ait, pour tous $\alpha$ et $\beta$ dans $K$, et tout entier naturel $n$ : $\delta_{n}(\alpha \beta)=\delta_{0}(\alpha) \Delta_{0}^{n}(\beta)+\delta_{1}(\alpha) \Delta_{1}^{n}(\beta)+\cdots+\delta_{n}(\alpha) \Delta_{n}^{n}(\beta)$, où la famille $\left(\Delta_{i}^{n}\right)_{n \geq 0 ; 0 \leq i \leq n}$ d'applications additives de $K$ dans $K$, dite famille des itérés de $S$, est définie par les relations de récurrence $\Delta_{0}^{n}=\delta_{n}$ et $\Delta_{i}^{n}=\delta_{0} \Delta_{i-1}^{n-1}+\delta_{1} \Delta_{i-1}^{n-2}+\delta_{2} \Delta_{i-1}^{n-3}+\cdots+\delta_{n-i} \Delta_{i-1}^{i-1}$ pour $1 \leq i \leq n$.

Si $S=\left(\delta_{n}\right)_{n \geq 0}$ est une haute dérivation de $K$, on peut munir l'ensemble des séries formelles en une indéterminée $X$, à coefficients dans $K$, d'une structure d'anneau, pour l'addition usuelle terme à terme, et pour le produit défini à partir de la relation de commutation: $X \alpha=\delta_{0}(\alpha) X+\delta_{1}(\alpha) X^{2}+\cdots+\delta_{n}(\alpha) X^{n+1}+\cdots$ pour tout $\alpha \in K$. L'anneau obtenu, noté $K[[X, S]]$, est un anneau de Cohen au sens de [11], c'est-à-dire un anneau de valuation discrète complet non commutatif admettant un sous-corps de représentants de son corps 
résiduel. Réciproquement, tout anneau de Cohen est de ce type (cf. [11] Théorème 1). Conformément à la définition donnée en [11], un élément $X^{\prime}=\alpha_{0}+\alpha_{1} X+\alpha_{2} X^{2}+\cdots$ de $A=K[[X, S]] \quad\left(\alpha_{i} \in K\right.$ pour tout i) est une uniformisante si et seulement si $\alpha_{0}=0, \alpha_{1} \neq 0$ et $\alpha_{1}$ appartient au centre $Z$ de $K$. Pour toute uniformisante $X^{\prime}$ de $A$, il existe une haute dérivation $S^{\prime}$ de $K$ telle que $A=K\left[\left[X^{\prime}, S^{\prime}\right]\right]$. Réciproquement, deux hautes dérivations données $S$ et $S^{\prime}$ de $K$ sont dites équivalentes si elles définissent sur $K$ le même anneau de Cohen, c'est-à-dire s'il existe dans l'anneau $A=K[[X, S]]$ une uniformisante $X^{\prime}$ telle que $A=K\left[\left[X^{\prime}, S^{\prime}\right]\right]$.

1.2. Hautes dérivations d'un corps de caractéristique nulle. Soit $K$ un corps gauche, de centre $Z$; il est bien connu (cf. [2], [10], [11], $\ldots$ ) que, pour toute dérivation $\delta$ de $K$, la suite $\left(\delta^{n}\right)_{n \geq 0}$ des puissances de composition de $\delta$ est une haute dérivation de $K$. Le corps des fractions de l'anneau de Cohen $K\left[\left[X,\left(\delta^{n}\right)_{n \geq 0}\right]\right]$ est alors simplement un corps d'opérateurs pseudo-différentiels formels (cf. [7]). Plus généralement, si $K$ est de caractéristique nulle, on a indiqué en [3], [5], [6] une méthode pour construire des hautes dérivations dont chaque terme est un polynôme différentiel formel suivant une même dérivation de $K$. Pour cela, à partir d'une dérivation $\delta$ de $K$ et d'une suite $a=\left(a_{n}\right)_{n \geq 1}$ d'éléments de $Z$, on définit (par des relations de récurrence complexes, figurant en [5] ou [6]) une famille $\left(b_{n, j}\right)_{n \geq 1 ; 1 \leq j \leq n}$ d'éléments de $Z$, et l'on pose $\delta_{0}=\mathrm{id}_{K}$ et, pour tout entier $n \geq 1, \delta_{n}=b_{n, 1} \delta+b_{n, 2} \delta^{2}+\cdots+b_{n, n} \delta^{n} \quad\left(\delta_{n}\right.$ est "sans terme constant", de degré inférieur ou égal à $n$, et, lorsque $\delta$ n'est pas intérieure, le coefficient du terme $\delta$ de degré 1 est $b_{n, 1}=a_{n}$ ). La suite $S=\left(\delta_{n}\right)_{n \geq 0}$ ainsi obtenue est une haute dérivation de $K$, dite monogène déterminée par $\delta$ et $a$. On trouvera en [3] la forme explicite des premiers termes de $S$, et en [6] tous les détails de la construction, que l'on ne peut reprendre ici. A noter cependant que si $a_{1}=1$ et $a_{n}=0$ pour tout $n \geq 2, S$ n'est autre que l'exemple $\left(\delta^{n}\right)_{n \geq 0}$ évoqué plus haut.

L'importance des hautes dérivations monogènes est mise en évidence par le théorème suivant, publié en [3] et démontré en [6]: si $K$ est un corps commutatif de caractéristique nulle, alors toute haute dérivation de $K$ est monogène. Lorsque $K$ n'est pas commutatif, il existe des hautes dérivations de $K$ qui ne sont pas monogènes (cf. [5] et [6]) et l'on reformule le problème sous la forme plus générale suivante: toute haute dérivation d'un corps gauche de caractéristique nulle est-elle équivalente à une haute dérivation monogène? Si tel était le cas, 
les hautes dérivations monogènes suffiraient à décrire tous les anneaux de Cohen $K[[X, S]]$ que l'on peut construire sur $K$, et l'on pourrait envisager de classer toutes les hautes dérivations de $K$ par un critère d'équivalence analogue au Théorème 1 de [4]. Rappelons les éléments de réponse au problème ci-dessus actuellement connus; pour cela, convenons d'appeler $Z$-haute dérivation de $K$ toute haute dérivation $\left(\delta_{n}\right)_{n \geq 0}$ de $K$ telle que $\delta_{n}(\alpha)=0$ pour tout $\alpha \in Z$ et tout $n \geq 1$. Désignons par $\operatorname{Der}_{Z} K$ le $Z$-espace vectoriel des $Z$-dérivations de $K$, par Int $K$ le sous-espace des dérivations intérieures de $K$, et par $\varepsilon_{K}$ la dimension (finie ou infinie) sur $Z$ du quotient $\operatorname{Der}_{Z} K / \operatorname{Int} K$. On vérifie aisément que $\varepsilon_{K}$ n'est autre que la dimension sur $Z$ du premier module de cohomologie de Hochschild $H^{1}(K, K)$ de $K$ (considéré comme $Z$-algèbre) à coefficients dans $K$ (considéré comme $K$-bimodule). Les principaux résultats de [5], démontrés en [6], sont alors: toute haute dérivation de $K$ qui n'est pas une $Z$-haute dérivation est équivalente à une haute dérivation monogène, de sorte qu'il suffit maintenant d'étudier les $Z$-hautes dérivations de $K$, pour lesquelles on a: si $\varepsilon_{K} \leq 1$, toute $Z$-haute dérivation de $K$, et donc toute haute dérivation de $K$, est équivalente à une haute dérivation monogène. Pour $\varepsilon_{K} \geq 2$, on ne sait pas, pour l'instant, résoudre le problème en toute généralité. La principale difficulté réside dans le fait qu'il semble alors nécessaire de connaître explicitement la structure des $Z$ dérivations de $K$ (question dont la résolution est rarement immédiate) avant d'envisager l'étude des $Z$-hautes dérivations. Il est cependant des exemples de corps pour lesquels on peut donner une réponse:

1.3. Le résultat principal. L'object de l'étude qui suit est de construire un corps non commutatif $K$ de caractéristique nulle, de centre $Z$, dont on sait décrire toutes les $Z$-dérivations, tel que $\varepsilon_{K}=2$, et dans lequel toute haute dérivation est équivalente à une haute dérivation monogène.

II. Construction du corps $K$. Soit $k$ un corps commutatif de caractéristique nulle, et $F$ un corps local commutatif de corps résiduel $k$. D'après le théorème de Cohen, pour tout choix d'une uniformisante $Y$ de $F, F$ s'identifie au corps de séries de Laurent $k((Y))$. Considérons dans $F$ la dérivation $\partial_{Y}$ par rapport à la variable $Y$, et construisons le corps d'opérateurs pseudo-différentiels formels $K=$ $F\left(\left(X, \partial_{Y}\right)\right)=k((Y))\left(\left(X, \partial_{Y}\right)\right)$. Le produit non commutatif dans $K$ est défini à partir de la relation de commutation: $X t=\sum_{i \geq 0} \partial_{Y}^{i}(t) X^{i+1}$ 
pour tout $t \in F$, que l'on peut écrire: $X t-t X=\left[\sum_{i \geq 1} \partial_{Y}^{i}(t) X^{i}\right] X=$ $X \partial_{Y}(t) X$, ou encore: $t X^{-1}-X^{-1} t=\partial_{Y}(t)$ pour tout $t \in F$. En prolongeant la dérivation $\partial_{Y}$ à $K$ par: $\partial_{Y}\left(\sum_{n} t_{n} X^{n}\right)=\sum_{n} \partial_{Y}\left(t_{n}\right) X^{n}$, [où $t_{n} \in F$ pour tout $n>-\infty$ ], on étend aisément la relation cidessus en:

$$
t X^{-1}-X^{-1} t=\partial_{Y}(t) \quad \text { pour tout } t \in K .
$$

En particulier: $Y X^{-1}-X^{-1} Y=1$; il en résulte par récurrence que, pour tout entier relatif $n, Y X^{-n}-X^{-n} Y=n X^{-n+1}=\partial_{X^{-1}}\left(X^{-n}\right)$, et finalement:

$$
Y t-t Y=\partial_{X^{-1}}(t) \quad \text { pour tout } t \in K .
$$

On déduit sans difficulté des relations $\left(^{*}\right)$ et $\left({ }^{* *}\right)$, ou encore du corollaire du Théorème 2 de [4], que le centre $Z$ de $K$ est le corps $k$. L'étude des $Z$-dérivations de $K$ repose donc sur la détermination des $k$-dérivations de $K$.

\section{Structure des $Z$-dérivations de $K$.}

3.1. Construction de dérivations non intérieures de $K$. Notons $|\cdot|$ la valeur absolue sur $K$ définie par la valuation discrète $o_{X}$ de $K$ et, pour tout opérateur $u$ de $K, Q$-linéaire et lipschitzien, $\|u\|=$ $\operatorname{Sup}_{t \in K ; t \neq 0}|u(t)| /|t|$ la norme ultramétrique associée. On désigne par $s_{X}$ (respectivement $s_{Y}$ ) l'automorphisme intérieur de $K$ déterminé par $X$ (respectivement $Y$ ), vérifiant donc $s_{X}(t)=X t X^{-1}$ (respectivement $\left.s_{Y}(t)=Y t Y^{-1}\right)$ pour tout $t \in K$. On peut remarquer aisément que, pour tout $t$ non nul de $K$, on a $o_{X}\left(s_{X}(t)-t\right)>o_{X}(t)$ et $o_{X}\left(s_{Y}(t)-t\right)>o_{X}(t)$, d'où $\left\|s_{X}-\mathrm{id}_{K}\right\|<1$ et $\left\|s_{Y}-\mathrm{id}_{K}\right\|<1$. En utilisant la Proposition 2 de [12], on construit alors deux dérivations:

$$
d_{X}=\sum_{n \geq 1}\left[(-1)^{n-1} / n\right]\left(s_{X}-\mathrm{id}_{K}\right)^{n}=\log \left(s_{X}\right)
$$

et

$$
d_{Y}=\sum_{n \geq 1}\left[(-1)^{n-1} / n\right]\left(s_{Y}-\mathrm{id}_{K}\right)^{n}=\log \left(s_{Y}\right) .
$$

Il est clair que:

$$
d_{X} \text { et } d_{Y} \text { sont des } k \text {-dérivations de } K \text {, et }
$$

$$
d_{X}(X)=d_{Y}(Y)=0 \text {. }
$$

Il résulte de la relation $\left({ }^{*}\right)$ du paragraphe II que, pour tout $t \in K$, $\left(s_{X}-\mathrm{id}_{K}\right)(t)=X t X^{-1}-t=X\left(\partial_{Y}(t)+X^{-1} t\right)-t=X \partial_{Y}(t)$. En 
particulier: $\left(s_{X}-\mathrm{id}_{K}\right)(Y)=X$ et $\left(s_{X}-\mathrm{id}_{K}\right)^{n}(Y)=0$ pour tout $n \geq 2$; d'où, avec (3.1.1):

$$
d_{X}(Y)=X
$$

On montre de même, avec $\left({ }^{* *}\right)$ :

$$
d_{Y}\left(X^{-1}\right)=Y^{-1} \text {. }
$$

Si $d_{X}$ était intérieure, il existerait $t \in K$ tel que $d_{X}=a d_{t}$. On aurait en particulier, avec (3.1.4), (3.1.5), (*) et $\left(^{* *}\right)$ : $0=d_{X}\left(X^{-1}\right)=$ $X^{-1} t-t X^{-1}=-\partial_{Y}(t)$ et $X=d_{X}(Y)=Y t-t Y=\partial_{X^{-1}}(t)$ d'où $t \in k((X))$ et $\partial_{X^{-1}}(t)=X$, ce qui est impossible; donc: la $k$-dérivation $d_{X}$ de $K$ n'est pas intérieure.

On établit de la même façon:

la $k$-dérivation $d_{Y}$ de $K$ n'est pas intérieure.

Il est clair enfin que $d_{X}$ et $d_{Y}$ sont linéairement indépendantes sur $k$, et l'on peut alors décrire toutes les $k$-dérivations de $K$ :

3.2. Théorème. Pour toute $k$-dérivation $d$ de $K$, il existe un unique couple $(a, b)$ d'éléments de $k$, et un élément $e$ de $K$, déterminé à un élément de $k$ près, tels que: $d=a \cdot d_{X}+b \cdot d_{Y}+a d_{e}$. Il en résulte que $\varepsilon_{k}=2$.

Preuve. Soit $d$ une $k$-dérivation quelconque de $K$. Désignons par $a \in k((Y))$ le coefficient de $X$ dans le développement de $d(Y)$ dans $K$ suivant les puissances de $X$. L'élément $d(Y)-a X$ est alors intégrable par rapport à $X^{-1}$, et il existe donc un élément $f$ de $K$, déterminé à un élément de $k((Y))$ près, tel que:

$$
d(Y)=a X+\partial_{X^{-1}}(f) .
$$

Par ailleurs, appliquons $d$ à la relation $Y X^{-1}-X^{-1} Y=1$ définisant le produit dans $K$; il vient: $d(Y) X^{-1}-X^{-1} d(Y)+Y d\left(X^{-1}\right)-$ $d\left(X^{-1}\right) Y=0$, soit, avec $\left(^{*}\right)$ et $\left(^{* *}\right)$ :

$$
\partial_{Y}[d(Y)]+\partial_{X^{-1}}\left[d\left(X^{-1}\right)\right]=0 .
$$

Reprenant (3.2.1), on obtient: $\partial_{X^{-1}}\left[d\left(X^{-1}\right)\right]=-\partial_{Y}(a) X-\partial_{Y} \partial_{X^{-1}}(f)$ $=-\partial_{Y}(a) X-\partial_{X^{-1}} \partial_{Y}(f)$, d'où $\partial_{Y}(a)=0$ et $\partial_{X^{-1}}\left[d\left(X^{-1}\right)+\partial_{Y}(f)\right]=$ 0 , et donc $a \in k$ et $d\left(X^{-1}\right)+\partial_{Y}(f) \in k((Y))$. Posons $b \in k$ le coefficient de $Y^{-1}$ dans le développement de $d\left(X^{-1}\right)+\partial_{Y}(f)$ dans $k((Y))$, de sorte qu'il existe $g \in k((Y))$, déterminé à un élément de $k$ 
près, tel que $d\left(X^{-1}\right)+\partial_{Y}(f)=b Y^{-1}+\partial_{Y}(g)$, c'est-à-dire $d\left(X^{-1}\right)=$ $b Y^{-1}-\partial_{Y}(f-g)$. En résumé, et en rappelant (3.2.1), on a montré qu'il existait, pour toute $k$-dérivation $d$ de $K$, un unique couple $(a, b)$ d'éléments de $k$ et un élémént $e=f-g$ de $K$, déterminé à un élément de $k$ près, tels que $d(Y)=a X+\partial_{X^{-1}}(e)$ et $d\left(X^{-1}\right)=$ $b Y^{-1}-\partial_{Y}(e)$. On en déduit, avec (3.1.4), (3.1.5), (3.1.6), $\left(^{*}\right)$ et $\left(^{* *}\right)$, que les $k$-dérivations $d$ et $a \cdot d_{X}+b \cdot d_{Y}+a d_{e}$ coïncident en $Y$ et en $X^{-1}$, et sont donc égales sur $K$. Il résulte alors de (3.1.7), (3.1.8) et de la $k$-linéaire indépendance de $d_{X}$ et $d_{Y}$, puisque $Z=k$, que $\varepsilon_{K}=2$.

\section{Structure des hautes dérivations de $K$.}

4.1. ThÉORÈme. Soit $K$ le corps gauche construit et étudié aux paragraphes précédents. Toute $Z$-haute dérivation de $K$, et donc toute haute dérivation de $K$, est équivalente à une haute dérivation monogène.

La démonstration, proposée plus loin en 4.5, repose sur deux lemmes. Le premier est un résultat technique sur certains changements d'uniformisantes.

4.2. Lemme. Soit $S=\left(\delta_{n}\right)_{n \geq 0}$ une haute dérivation de $K$. On désigne par $A$ l'anneau de Cohen $K[[V, S]]$, en l'indéterminée $V$. On note $(V)$ l'idéal maximal de $A$. Soient $V^{\prime}$ et $V^{\prime \prime}$ deux uniformisantes de $A$, de hautes dérivations associées respectives $S^{\prime}=$ $\left(\delta_{n}^{\prime}\right)_{n \geq 0}$ et $S^{\prime \prime}=\left(\dot{\delta}_{n}^{\prime \prime}\right)_{n \geq 0}$; on a les deux assertions suivantes:

(i) s'il existe un entier $m \geq 1$ tel que $V^{\prime}-V^{\prime \prime} \in(V)^{m+1}$, alors $\delta_{n}^{\prime}=\delta_{n}^{\prime \prime}$ pour tout entier naturel $n \leq m-1$.

(ii) s'il existe un entier $m \geq 1$ et un élément $u$ de $K$ tels que $V^{\prime}=V+u V^{m+1}$, alors $\delta_{n}^{\prime}=\delta_{n}$ pour tout entier naturel $n \leq m-1$ et $\delta_{m}^{\prime}=\delta_{m}-a d_{u}$.

Preuve. Soit $T=V^{\prime}-V^{\prime \prime} \in(V)^{m+1}$. Pour tout $\alpha \in K$, on a d'une part $V^{\prime \prime} \alpha=\sum_{n \geq 0} \delta_{n}^{\prime \prime}(\alpha) V^{\prime \prime n+1}$, et d'autre part

$$
V^{\prime \prime} \alpha=\left(V^{\prime}-T\right) \alpha=\sum_{n \geq 0} \delta_{n}^{\prime}(\alpha) V^{\prime n+1}-T \alpha .
$$

Or, pour tout $n \geq 0$,

$$
V^{\prime n+1}=\left(V^{\prime \prime}+T\right)^{n+1}=V^{\prime \prime n+1}+T_{n}
$$


où $T_{n} \in(V)^{m+1}$. Comme $T \alpha \in(V)^{m+1}$, on peut donc écrire $V^{\prime \prime} \alpha=$ $\sum_{n \geq 0} \delta_{n}^{\prime}(\alpha) V^{\prime \prime n+1}+U$, avec $U \in(V)^{m+1}$. En identifiant les deux développements suivant les puissances de $V^{\prime \prime}$, on obtient $\delta_{n}^{\prime \prime}=\delta_{n}^{\prime}$ pour tout $n \leq m-1$, ce qui établit la première assertion. Choisissons maintenant $V^{\prime \prime}=V$ et $V^{\prime}=V+u V^{m+1}$, où $u \in K$, de sorte que, pour $n \geq 1, V^{\prime n+1}=\left(V+u V^{m+1}\right)^{n+1}=V^{n+1}+T_{n}$ avec $T_{n} \in(V)^{m+2}$. De plus $T \alpha=u V^{m+1} \alpha=u \alpha V^{m+1}+T^{\prime}$ avec $T^{\prime} \in(V)^{m+2}$. On peut alors reprendre le développement de $V^{\prime \prime} \alpha$ sous la forme $\delta_{0}^{\prime}(\alpha)\left(V+u V^{m+1}\right)+\sum_{n \geq 1} \delta_{n}^{\prime}(\alpha) V^{n+1}-u \alpha V^{m+1}+U$ où $U \in(V)^{m+2}$. En rappelant que $\delta_{0}^{\prime}=\mathrm{id}_{K}$, le coefficient du terme $V^{m+1} \mathrm{du}$ développement est $\alpha u+\delta_{m}^{\prime}(\alpha)-u \alpha$, d'où le résultat. A noter que cette démonstration reste valable si l'on remplace $K$ par un corps gauche quelconque.

En vue du second lemme, rappelons les éléments suivants:

4.3. Définition et notation. Soit $S=\left(\delta_{n}\right)_{n \geq 0}$ une haute dérivation de $K$, distincte de $\left(\operatorname{id}_{K}, 0,0, \ldots, 0, \ldots\right)$. On appelle ordre de $S$ l'entier $p \geq 1$ tel que $\delta_{i}=0$ pour $0<i<p$ et $\delta_{p} \neq 0$. L'application $\delta_{p}$ est alors une dérivation de $K$. Par ailleurs, pour toute dérivation $\delta$ de $K$ et toute suite $a=\left(a_{n}\right)_{n \geq 1}$ d'éléments du centre $Z$ de $K$, on note $H D(\delta, a)$ la haute dérivation monogène déterminée par $\delta$ et $a$. Si $\delta \neq 0$, l'ordre de $H D(\delta, a)$ est celui de la suite $a$.

4.4. LEMME. Considérons le corps $K=k((Y))\left(\left(X, \partial_{Y}\right)\right)$ construit et étudié aux paragraphes précédents. Soient $T=\left(d_{n}\right)_{n \geq 0}$ et $T^{\prime}=$ $\left(d_{n}^{\prime}\right)_{n \geq 0}$ deux $k$-hautes dérivations de $K$, de même ordre $p$. Supposons qu'il existe un entier $n \geq p+1$ tel que $d_{i}=d_{i}^{\prime}$ pour tout $i \leq n-1$, et que la $k$-dérivation $d_{p}$ soit de la forme $d_{p}=\alpha \cdot d_{X}+\beta \cdot d_{Y}$ (avec $\alpha \in k$ et $\beta \in k)$. Alors il existe un élément $c$ de $k$ et un élément $e$ de $K$ tels que $d_{n}-d_{n}^{\prime}=c \cdot d_{p}+a d_{e}$.

Preuve. Avec les notations et hypothèses du lemme, on note $D_{i}^{j}$ (respectivement $D_{i}^{\prime j}$ ) le terme général de la famille des itérés de $T$ (respectivement $T^{\prime}$ ). Soient $x$ et $y$ deux élément quelconques de $K$. Par définition d'une haute dérivation, on a:

$$
d_{n+p}(x y)=\sum_{i=0}^{n+p} d_{i}(x) D_{i}^{n+p}(y) \text {. }
$$

Mais $T$ est d'ordre $p$, d'où $d_{i}=0$ si $1 \leq i \leq p-1$; à l'aide des relations de récurrence définissant les applications $D_{i}^{j}$, on vérifie aussi 
que $D_{i}^{n+p}=0$ si $1 \leq n+p-i \leq p-1$, c'est-à-dire si $n+1 \leq i \leq$ $n+p-1$. La relation (4.4.1) s'écrit:

$$
d_{n+p}(x y)=x d_{n+p}(y)+\sum_{i=p}^{n} d_{i}(x) D_{i}^{n+p}(y)+d_{n+p}(x) y .
$$

De même:

$$
d_{n+p}^{\prime}(x y)=x d_{n+p}^{\prime}(y)+\sum_{i=p}^{n} d_{i}^{\prime}(x) D_{i}^{\prime n+p}(y)+d_{n+p}^{\prime}(x) y .
$$

Or $d_{i}=d_{i}^{\prime}$ si $i \leq n-1$, et donc $D_{i}^{n+p}=D_{i}^{\prime n+p}$ si $i \geq p+1$; la différence membre à membre de (4.4.2) et (4.4.3) conduit alors à:

(4.4.4) $\left(d_{n+p}-d_{n+p}^{\prime}\right)(x y)$

$$
\begin{aligned}
= & x\left(d_{n+p}-d_{n+p}^{\prime}\right)(y)+\left(d_{n+p}-d_{n+p}^{\prime}\right)(x) y \\
& +d_{p}(x)\left(D_{p}^{n+p}-D_{p}^{\prime n+p}\right)(y)+\left(d_{n}-d_{n}^{\prime}\right) D_{n}^{n+p}(y) .
\end{aligned}
$$

Un calcul annexe permet d'établir $D_{n}^{n+p}=(n+1) d_{p}$ et $D_{p}^{n+p}-D_{p}^{\prime n+p}=$ $(p+1)\left(d_{n}-d_{n}^{\prime}\right)$. Posons enfin $d=d_{n}-d_{n}^{\prime}$. (4.4.4) devient:

$$
\begin{aligned}
\left(d_{n+p}-\right. & \left.d_{n+p}^{\prime}\right)(x y) \\
= & x\left(d_{n+p}-d_{n+p}^{\prime}\right)(y)+\left(d_{n+p}-d_{n+p}^{\prime}\right)(x) y \\
& +(p+1) d_{p}(x) d(y)+(n+1) d(x) d_{p}(y) .
\end{aligned}
$$

Par ailleurs,

$$
d_{n}(x y)=\sum_{i=0}^{n} d_{i}(x) D_{i}^{n}(y)=x d_{n}(y)+\sum_{i=p}^{n-1} d_{i}(x) D_{i}^{n}(y)+d_{n}(x) y
$$

et

$$
d_{n}^{\prime}(x y)=x d_{n}^{\prime}(y)+\sum_{i=p}^{n-1} d_{i}^{\prime}(x) D_{i}^{\prime n}(y)+d_{i}^{\prime}(x) y .
$$

Comme, pour $p \leq i<n, d_{i}=d_{i}^{\prime}$ et $D_{i}^{n}=D_{i}^{\prime n}$, on obtient: $\left(d_{n}-d_{n}^{\prime}\right)(x y)=x\left(d_{n}-d_{n}^{\prime}\right)(y)+\left(d_{n}-d_{n}^{\prime}\right)(x) y$, de sorte que $d=d_{n}-d_{n}^{\prime}$ est une dérivation de $K$ (et même une $k$-dérivation puisque $T$ et $T^{\prime}$ sont des $k$-hautes dérivations). Il existe donc, d'après le Théorème 3.2 ci-dessus, $a \in k, b \in k$ et $e \in K$ tels que $d=a \cdot d_{X}+b \cdot d_{Y}+a d_{e}$, d'où, avec (3.1.5), (3.1.6), (*) et $\left(^{* *}\right): d(Y)=a X+\partial_{X^{-1}}(e)$ et $d\left(X^{-1}\right)=b Y^{-1}-\partial_{Y}(e)$. De même, $d_{p}=\alpha \cdot d_{X}+\beta \cdot d_{Y}$ par hypothèse, d'où $d_{p}(Y)=\alpha X$ et $d_{p}\left(X^{-1}\right)=\beta Y^{-1}$. Dès lors, si l'on applique $d_{n+p}-d_{n+p}^{\prime}$ aux deux membres de l'égalité $Y X^{-1}-X^{-1} Y=1$ 
définissant le produit dans $K$, on obtient, grâce à (4.4.5):

$$
\begin{aligned}
& Y\left(d_{n+p}-d_{n+p}^{\prime}\right)\left(X^{-1}\right)+\left(d_{n+p}-d_{n+p}^{\prime}\right)(Y) X^{-1} \\
& \quad+(p+1) \alpha b X Y^{-1}-(p+1) \alpha X \partial_{Y}(e)+(n+1) a \beta X Y^{-1} \\
& \quad+(n+1) \beta \partial_{X^{-1}}(e) Y^{-1}-X^{-1}\left(d_{n+p}-d_{n+p}^{\prime}\right)(Y) \\
& \quad-\left(d_{n+p}-d_{n+p}^{\prime}\right)\left(X^{-1}\right) Y-(p+1) \beta a Y^{-1} X \\
& \quad-(p+1) \beta Y^{-1} \partial_{X^{-1}}(e)-(n+1) b \alpha Y^{-1} X \\
& \quad+(n+1) \alpha \partial_{Y}(e) X=0,
\end{aligned}
$$

qui devient, avec $\left({ }^{*}\right)$ et $\left({ }^{* *}\right)$ :

$$
\begin{aligned}
\partial_{X^{-1}} & {\left[\left(d_{n+p}-d_{n+p}^{\prime}\right)\left(X^{-1}\right)\right] } \\
& +\partial_{Y}\left[\left(d_{n+p}-d_{n+p}^{\prime}\right)(Y)+(n+1) \alpha e X-(p+1) \alpha X e\right] \\
= & -(p+1) \alpha b X Y^{-1}-(n+1) a \beta X Y^{-1}-(n+1) \beta \partial_{X^{-1}}(e) Y^{-1} \\
& +(p+1) \beta a Y^{-1} X+(p+1) \beta Y^{-1} \partial_{X^{-1}}(e)+(n+1) b \alpha Y^{-1} X .
\end{aligned}
$$

Le coefficient de $X$ dans le développement suivant les puissances de $X$ dans $K$ du second membre est: $(n+1)(b \alpha-a \beta) Y^{-1}$ $(p+1)(\alpha b-\beta a) Y^{-1}$. Dans le premier membre, ce coefficient est nul dans le premier terme, et de la forme $\partial_{Y}(t)$ dans le second terme [avec $t \in k((Y))]$. En identifiant, on en déduit que: $\partial_{Y}(t)=$ $(n-p)(b \alpha-a \beta) Y^{-1}$, et donc, puisque $n-p>0, b \alpha=a \beta$. Si $\alpha=0$, alors, comme $d_{p} \neq 0, \beta \neq 0$ et $a=0$; on pose $c=b / \beta$. Si $\beta=0, b=0$ et l'on prend $c=a / \alpha$. Si $\alpha \neq 0$ et $\beta \neq 0, c=$ $a / \alpha=b / \beta$. Dans les trois cas, on a $d=c \cdot d_{p}+a d_{e}$, ce qui achève la démonstration du lemme.

4.5. Démonstration du Théorème 4.1. Puisque le centre $Z$ de $K$ est $k$, il suffit de montrer le théorème pour les $k$-hautes dérivations. Soit $S=\left(\delta_{n}\right)_{n \geq 0}$ une telle $k$-haute dérivation. $\mathrm{Si}$

$$
S=\left(\mathrm{id}_{K}, 0,0, \ldots, 0, \ldots\right),
$$

$S$ est monogène, déterminée par la dérivation nulle et une suite $a$ quelconque d'éléments de $k$. Sinon, soit $p$ l'ordre de $S$. La dérivation $\delta_{p}$ est une $k$-dérivation de $K$; en appliquant le Théorème 3.2, il existe $\alpha \in k, \beta \in k$ et $e \in K$ tels que $\delta_{p}=\alpha \cdot d_{X}+\beta \cdot d_{Y}+a d_{e}$. D'après le Lemme 4.2(ii), $S$ est équivalente à une haute dérivation $S_{1}=\left(\delta_{1, n}\right)_{n \geq 0}$ d'ordre $p$ telle que $\delta_{1, p}=\alpha \cdot d_{X}+\beta \cdot d_{Y}$. On a montré en [6] que, dans un corps gauche quelconque de caractéristique nulle, toute haute dérivation équivalente à une $Z$-haute dérivation est 
elle-même une $Z$-haute dérivation. Donc ici, $S_{1}$ est une $k$-haute dérivation de $K$. Appelons $\delta$ la $k$-dérivation $\alpha \cdot d_{X}+\beta \cdot d_{Y}$.

Soit $a_{1}=\left(a_{1, i}\right)_{i \geq 1}$ la suite d'éléments de $k$ définie par $a_{1, p}=1$ et $a_{1, i}=0$ si $i \neq p$. Posons $T_{1}=H D\left(\delta, a_{1}\right)=\left(d_{1, n}\right)_{n \geq 0}$. On a $d_{1, i}=\delta_{1, i}$ pour tout $i \leq p$. Appliquons le Lemme 4.4 avec $T=S_{1}$, $T^{\prime}=T_{1}$ et $n=p+1$. Il existe $b_{p+1} \in k$ et $e_{p+2} \in K$ tels que $\delta_{1, p+1}=d_{1, p+1}+b_{p+1} \delta+a d_{e_{p+2}}$. La dérivation $\delta$ n'étant pas intérieure, le coefficient de $\delta$ dans $d_{1, p+1}$ est $a_{1, p+1}=0$, et donc le coefficient de $\delta$ dans le polynôme différentiel formel $d_{1, p+1}+b_{p+1} \delta$ est $b_{p+1}$.

Soient alors, dans l'anneau de Cohen $A=K\left[\left[X_{1}, S_{1}\right]\right]$, l'uniformisante $X_{2}=X_{1}+e_{p+2} X_{1}^{p+2}$, et $S_{2}=\left(\delta_{2, n}\right)_{n \geq 0}$ la $k$-haute dérivation associée. D'après le Lemme $4.2(\mathrm{ii}), \delta_{2, i}=\delta_{1, i}$ pour $i \leq p$, et $\delta_{2, p+1}=d_{1, p+1}+b_{p+1} \delta$. On désigne par $a_{2}=\left(a_{2, i}\right)_{i \geq 1}$ la suite d'éléments de $k$ définie par $a_{2, p}=1, a_{2, p+1}=b_{p+1}$ et $a_{2, i}=0$ sinon. Soit $T_{2}=H D\left(\delta, a_{2}\right)=\left(d_{2, n}\right)_{n \geq 0}$. Comme $a_{1, j}=a_{2, j}$ pour tout $j \leq p$, et comme $a_{1, p+1}=0$ alors que $a_{2, p+1}=b_{p+1}$, on a $d_{1, j}=d_{2, j}$ pour tout $j \leq p$, et $d_{2, p+1}=d_{1, p+1}+b_{p+1} \delta$. Il en résulte que $\delta_{2, i}=d_{2, i}$ pour tout $i \leq p+1$. Appliquons alors le Lemme 4.4 avec $T=S_{2}, T^{\prime}=T_{2}$ et $n=p+2$. Il existe $b_{p+2} \in k$ et $e_{p+3} \in K$ tels que $\delta_{2, p+2}=d_{2, p+2}+b_{p+2} \delta+a d_{e_{p+3}}$.

Soient alors dans $A$ l'uniformisante $X_{3}=X_{2}+e_{p+3} X_{2}^{p+3}$ et $S_{3}=$ $\left(\delta_{3, n}\right)_{n \geq 0}$ la $k$-haute dérivation associée. D'après le Lemme 4.2(ii), $\delta_{3, i}=\delta_{2, i}$ pour tout $i \leq p+1$, et $\delta_{3, p+2}=d_{3, p+2}+b_{p+2} \delta$. Le coefficient de $\delta$ dans $d_{2, p+2}$ est $a_{2, p+2}=0$, donc le coefficient de $\delta$ dans le polynôme différentiel formel $\delta_{3, p+2}$ est $b_{p+2}$. On désigne par $a_{3}=\left(a_{3, i}\right)_{i \geq 1}$ la suite d'éléments de $k$ définie par $a_{3, p}=1, a_{3, p+1}=$ $b_{p+1}, a_{3, p+2}=b_{p+2}$, et $a_{3, i}=0$ sinon. Soit $T_{3}=H D\left(\delta, a_{3}\right)=$ $\left(d_{3, n}\right)_{n \geq 0}$. Comme $a_{2, j}=a_{3, j}$ pour tout $j \leq p+1$, et comme $a_{2, p+2}=0$ alors que $a_{3, p+2}=b_{p+2}$, on a $d_{3, j}=d_{2, j}$ pour $j \leq p+1$, et $d_{3, p+2}=d_{2, p+2}+b_{p+2} \delta$. Il en résulte que $\delta_{3, i}=d_{3, i}$ pour tout $i \leq p+2$. On applique ensuite le Lemme 4.4 avec $T=S_{3}, T^{\prime}=T_{3}$ et $n=p+3$.

En réitérant, on construit ainsi par récurrence:

- une suite $b=\left(b_{i}\right)_{i \geq 1}$ d'éléments de $k$, d'ordre $p$, avec $b_{p}=1$;

- une suite $\left(X_{n}\right)_{n \geq 1}$ d'uniformisantes de $A$, définie à partir d'une suite $\left(e_{i}\right)_{i \geq p+2}$ d'éléments de $K$ par la relation:

$$
X_{n}=X_{n-1}+e_{p+n} X_{n-1}^{p+n} \quad \text { pour tout } n \geq 2,
$$

telles que, si l'on appelle, pour tout $n \geq 1$ :

- $S_{n}=\left(\delta_{n, i}\right)_{i \geq 0}$ la $k$-haute dérivation associée à $X_{n}$, et 
- $T_{n}=\left(d_{n, i}\right)_{i \geq 0}$ la $k$-haute dérivation $H D\left(\delta, a_{n}\right)$ où $a_{n}=$ $\left(a_{n, i}\right)_{i \geq 0}$ vérifie:

$$
\begin{cases}a_{n, i}=b_{i} & \text { si } p \leq i<p+n \\ a_{n, i}=0 & \text { sinon }\end{cases}
$$

alors

$$
\delta_{n, i}=d_{n, i} \text { pour tout } i<p+n .
$$

Posons $T=H D(\delta, b)=\left(d_{i}\right)_{i \geq 0}$. Il résulte de (4.5.2) que:

$$
d_{i}=d_{n, i} \quad \text { pour tout } n \geq 1 \text { et tout } i<p+n \text {. }
$$

Par ailleurs, notons, pour tout $n \geq 1, X_{n}=\sum_{i \geq 1} x_{n, i} X_{1}^{i} \quad\left(x_{n, i} \in\right.$ $\left.K ; x_{n, 1}=1\right)$. L'égalité (4.5.1) se traduit par:

$$
x_{n, i}=x_{n-1, i} \quad \text { pour tout } n \geq 2 \text { et tout } i<p+n .
$$

Construisons une suite $\left(\bar{x}_{n}\right)_{n \geq 1}$ d'éléments de $K$ en posant: $\bar{x}_{1}=1$, $\bar{x}_{2}=\bar{x}_{3}=\cdots=\bar{x}_{p}=0$ (ceci lorsque $p>1$ ), et $\bar{x}_{p+i}=x_{i, p+i}$ pour tout $i \geq 1$; d'où l'on déduit, avec (4.5.5):

$$
\bar{x}_{i}=x_{n, i} \quad \text { pour tout } n \geq 1 \text { et tout } i \leq p+n .
$$

L'uniformisante $\bar{X}=\sum_{i \geq 1} \bar{x}_{i} X_{1}^{i}$ de $A$ est la limite de la suite de Cauchy $\left(X_{n}\right)_{n \geq 1}$, et l'on désigne par $\bar{S}=\left(\bar{\delta}_{n}\right)_{n \geq 0}$ la $k$-haute dérivation associée, qui est, par construction, équivalente à $S$. Appliquons le Lemme $4.2(\mathrm{i})$ avec $m=p+n \geq 2 ;(4.5 .6)$ conduit à: $\bar{\delta}_{i}=\delta_{n, i}$ pour tout $n \geq 1$ et tout $i \leq p+n-1$, ou encore, avec (4.5.3) et (4.5.4): $\bar{\delta}_{i}=d_{i}$ pour tout $n \geq 1$ et tout $i \leq p+n-1$. On conclut que $\bar{S}=T$, et donc que $S$ est équivalente à la $k$-haute dérivation monogène $T$, ce qui achève la démonstration.

\section{REFERENCES}

[1] H. H. Brungs and G. Törner, Skew power series rings and derivations, J. Algebra, 87 (1984), 368-379.

[2] P. M. Cohn, Free Rings and Their Relations, second edition, Academic Press, 1985.

[3] F. Dumas, Hautes dérivations et anneaux de séries formelles non commutatifs en caractéristique nulle, C.R. Acad. Sci. Paris, 303, série I, (1986), 383-385.

[4] _ Hautes dérivations équivalentes, en caractéristique nulle, C.R. Acad. Sci. Paris, 303, série I, (1986), 841-843.

[5] _- Hautes dérivations dans les corps gauches, en caractéristique nulle, C.R. Acad. Sci. Paris, 306, série I, (1988), 519-521.

[6] _- Corps locaux non commutatifs et hautes dérivations, Thèse (Université Blaise Pascal), 1988. 
[7] K. R. Goodearl, Centralizers in differential, pseudo-differential, and fractional differential operator rings, Rocky Mountain J. Math., 13, no. 4, (1983), 573618.

[8] B. Roux, Anneaux non commutatifs de valuation discrète ou finie, scindés, I et II, C.R. Acad. Sci. Paris, 302, série I, (1986), 259-262 et 291-293.

[9] __ Anneaux non commutatifs de valuation discrète ou finie, Cahiers Mathématiques de Montpellier, no. 36, 1986.

[10] __ Anneaux non commutatifs de valuation discrète, scindés, en caractéristique zéro, C.R. Acad. Sci. Paris, 303, série I, (1986), 663-666.

[11] R. Vidal, Anneaux de valuation discrète complets non commutatifs, Trans. Amer. Math. Soc., 267, 1, (1981), 65-81.

[12] __ Automorphismes et dérivations de "petites normes" sur un corps valué non archimédien, Ann. Sci. Univ. Clermont II, Sér. Math., Fasc. 21, (1982), 87-95.

Received October 12, 1990 and in revised form March 13, 1991.

UNIVERSITÉ DE REIMS

DÉPARTEMENT DE MATHÉMATIQUES

MOULIN DE LA HOUSSE

BP 347, 51062 Reims Cedex, France

\section{AND}

Université Blaise Pascal

CompleXe SCIentifique des Cézeaux

BP 45, 63170 Aubiere, France 


\section{PACIFIC JOURNAL OF MATHEMATICS EDITORS}

\author{
V. S. VARADARAJAN \\ (Managing Editor) \\ University of California \\ Los Angeles, CA 90024-1555-05 \\ Herbert Clemens \\ University of Utah \\ Salt Lake City, UT 84112 \\ F. Michael Christ \\ University of California \\ Los Angeles, CA 90024-1555 \\ THOMAS ENRIGHT \\ University of California, San Diego \\ La Jolla, CA 92093
}

Nicholas ERCOLANI

University of Arizona

Tucson, AZ 85721

R. FINN

Stanford University

Stanford, CA 94305

VAUGHAN F. R. JONES

University of California

Berkeley, CA 94720

Steven Kerckhoff

Stanford University

Stanford, CA 94305

\section{C. MOORE}

University of California

Berkeley, CA 94720

Martin ScharlemanN

University of California

Santa Barbara, CA 93106

HAROLD STARK

University of California, San Diego

La Jolla, CA 92093

\section{R. ARENS \\ ASSOCIATE EDITORS}

\begin{tabular}{|c|c|c|c|c|}
\hline R. ARENS & $\begin{array}{l}\text { E. F. BECKENBACH } \\
(1906-1982)\end{array}$ & B. H. NeumanN & $\begin{array}{c}\text { F. WolF } \\
(1904-1989)\end{array}$ & K. YoshidA \\
\hline \multicolumn{5}{|c|}{ SUPPORTING INSTITUTIONS } \\
\hline \multicolumn{2}{|c|}{ UNIVERSITY OF ARIZONA } & UNIVER: & OF OREG & \\
\hline \multicolumn{2}{|c|}{ UNIVERSITY OF BRITISH COLUMB } & & & \\
\hline \multicolumn{2}{|c|}{ CALIFORNIA INSTITUTE OF TECHNOLOGY } & OGY STANI & INIV & \\
\hline \multicolumn{2}{|c|}{ UNIVERSITY OF CALIFORNIA } & UNI & & \\
\hline \multicolumn{2}{|c|}{ MONTANA STATE UNIVERSITY } & UNIVE & OF T & \\
\hline \multirow{2}{*}{\multicolumn{2}{|c|}{ NIVERSITY OF NEVADA, RENO }} & UNIVE & OF U & \\
\hline & & WASH & N ST & ERSIT \\
\hline \multicolumn{2}{|c|}{$\begin{array}{l}\text { NEW MEXICO STATE UNIVERSITY } \\
\text { OREGON STATE UNIVERSITY }\end{array}$} & UNIVERS & OF WASHI & \\
\hline
\end{tabular}

The Supporting Institutions listed above contribute to the cost of publication of this Journal, but they are not owners or publishers and have no responsibility for its content or policies.

Mathematical papers intended for publication in the Pacific Journal of Mathematics should be in typed form or offset-reproduced (not dittoed), double spaced with large margins. Please do not use built up fractions in the text of the manuscript. However, you may use them in the displayed equations. Underline Greek letters in red, German in green, and script in blue. The first paragraph must be capable of being used separately as a synopsis of the entire paper. In particular it should contain no bibliographic references. Please propose a heading for the odd numbered pages of less than 35 characters. Manuscripts, in triplicate, may be sent to any one of the editors. Please classify according to the 1991 Mathematics Subject Classification scheme which can be found in the December index volumes of Mathematical Reviews. Supply name and address of author to whom proofs should be sent. All other communications should be addressed to the managing editor, or Elaine Barth, University of California, Los Angeles, California 90024-1555-05.

There are page-charges associated with articles appearing in the Pacific Journal of Mathematics. These charges are expected to be paid by the author's University, Government Agency or Company. If the author or authors do not have access to such Institutional support these charges are waived. Single authors will receive 50 free reprints; joint authors will receive a total of 100 free reprints. Additional copies may be obtained at cost in multiples of 50 .

The Pacific Journal of Mathematics (ISSN 0030-8730) is published monthly except for July and August. Regular subscription rate: $\$ 190.00$ a year (10 issues). Special rate: $\$ 95.00$ a year to individual members of supporting institutions.

Subscriptions, orders for numbers issued in the last three calendar years, and changes of address should be sent to Pacific Journal of Mathematics, P.O. Box 969, Carmel Valley, CA 93924, U.S.A. Old back numbers obtainable from Kraus Periodicals Co., Route 100, Millwood, NY 10546.

The Pacific Journal of Mathematics at P.O. Box 969, Carmel Valley, CA 93924 (ISSN 0030-8730) is published monthly except for July and August. Second-class postage paid at Carmel Valley, California 93924, and additional mailing offices. Postmaster: send address changes to Pacific Journal of Mathematics, P.O. Box 969, Carmel Valley, CA 93924.

PUBLISHED BY PACIFIC JOURNAL OF MATHEMATICS, A NON-PROFIT CORPORATION Copyright (C) 1992 by Pacific Journal of Mathematics 


\section{Pacific Journal of Mathematics}

Vol. 153, No. $2 \quad$ April, 1992

R. Ayala, Eladio Domínguez Murillo, Alberto Márquez Pérez and A.

Quintero, Lusternik-Schnirelmann invariants in proper homotopy

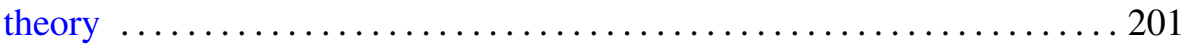

Hari Bercovici and Dan-Virgil Voiculescu, Lévy-Hinčin type theorems for multiplicative and additive free convolution $\ldots \ldots \ldots \ldots \ldots \ldots \ldots 217$

L. J. Bunce and Cho-Ho Chu, Compact operations, multipliers and Radon-Nikodým property in $J B^{*}$-triples $\ldots \ldots \ldots \ldots \ldots \ldots \ldots \ldots \ldots \ldots \ldots \ldots$

Marius Dadarlat, Gabriel Nagy, András Némethi and Cornel Pasnicu, Reduction of topological stable rank in inductive limits of $C^{*}$-algebras

François Dumas and Robert Vidal, Dérivations, et hautes dérivations, dans certains corps gauches de series de Laurent .................... 277

Mourad Ismail and Xin Li, On sieved orthogonal polynomials. IX:

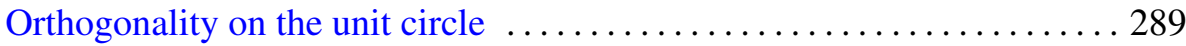

X. T. Liang and Y. W. Lu, A Phragmén-Lindelöf theorem ...............299

Mark Stephen Reeder, On certain Iwahori invariants in the unramified principal series

Shohei Tanaka, On the representation of the determinant of Harish-Chandra's $C$-function of $\operatorname{SL}(n, \mathbb{R})$

Fritz von Haeseler and Guentcho Svetoslavov Skordev, Borsuk-Ulam theorem, fixed point index and chain approximations for maps with multiplicity 